\title{
A BESSEL FUNCTION INEQUALITY CONNECTED WITH STABILITY OF LEAST SQUARE SMOOTHING
}

\author{
LEE LORCH ${ }^{1}$ AND PETER SZEGO
}

1. Introduction. In considering the stability (i.e., the asymptotic smoothness of higher order iterates) of continuous smoothing problems in least square approximation, H. S. Wilf $[7(\mathrm{a})]$ introduces a certain inequality ((2) below) involving Bessel functions.

His argument in support of this inequality requires correction [7(b)], which it is our purpose here to supply.

2. The inequality. Defining

$$
h_{\nu \lambda}(\theta)=1-\frac{\int_{0}^{\theta} t^{-\lambda} J_{\nu}(t) d t}{\int_{0}^{\infty} t^{-\lambda} J_{\nu}(t) d t},
$$

where $J_{\nu}(t)$ is the Bessel function of first kind and order $\nu$, the inequality in question is

$$
-1<h_{\nu \lambda}(\theta)<1 \quad(\theta \neq 0),
$$

for $\lambda=1 / 2, \nu=2 k+3 / 2, k$ a sufficiently large positive integer.

3. Preliminaries. In verifying (2) for appropriate $\lambda$ and $\nu$, some preliminary results will be needed. The first is a corrected version of Wilf's formula (8), which we establish in a somewhat extended form:

$$
\begin{aligned}
\lim _{\nu \rightarrow \infty} \frac{\nu^{\lambda} \int_{0}^{j_{\nu 1}} t^{-\lambda} J_{\nu}(t) d t}{\nu^{\lambda} \int_{0}^{\infty} t^{-\lambda} J_{\nu}(t) d t} & =\frac{1}{3}+\frac{1}{3} \int_{0}^{c}\left[J_{1 / 3}(t)+J_{-1 / 3}(t)\right] d t \\
& \doteq 1.2743521,
\end{aligned}
$$

where $\lambda>-1 / 2, j_{\nu 1}$ is the first positive zero of $J_{\nu}(t)$, and $c$ is the least positive zero of $J_{1 / 3}(t)+J_{-1 / 3}(t)$.

PROOF OF (3). The denominator of the first member of (3) is equal to $(\nu / 2)^{\lambda} \Gamma[(\nu+1-\lambda) / 2] / \Gamma[(\nu+1+\lambda) / 2]$ (cf., e.g., $[4$, p. $\left.414(11)]\right)$,

Received by the editors February 23, 1965.

1 This note was written while the first-named author was enjoying the hospitality of the Mathematics Institute, Aarhus University, Denmark. 
and so has limit equal to 1 . The limit of the numerator is equal to the subsequent members of (3) by [4, p. 409 (4)]. ${ }^{2}$

Another result to be used is

$$
\int_{0}^{j_{\nu 2}} t^{-\lambda} J_{\nu}(t) d t>0 \quad\left\{\begin{array}{cl}
\text { (i) } & \lambda>0, \quad \nu>-1 \text { or } \\
\text { (ii) })^{4} & \lambda>-1 / 2, \nu>1 / 2,
\end{array}\right.
$$

with $\lambda<\nu+1$ (to insure convergence of the integral at the origin) where $j_{\nu 2}$ is the second positive zero of $J_{\nu}(t)$.

Proof of (4)(i). For small positive $\epsilon$, the second mean-value theorem applies so that

$$
\begin{aligned}
\int_{0}^{j_{\nu 2}} t^{-\lambda} J_{\nu}(t) d t & >\int_{\epsilon}^{j_{\nu 2}} t^{-\lambda} J_{\nu}(t) d t, \\
& =\epsilon^{-\lambda} \int_{e}^{\eta} J_{\nu}(t) d t, \quad \epsilon<\eta<j_{\nu 2} .
\end{aligned}
$$

If $\eta \leqq j_{\nu 1}$, then this last integral is positive for $\nu>-1$, and (4)(i) is proved. If $\eta>j_{v 1}$, then this last integral clearly exceeds

$$
\epsilon^{-\lambda} \int_{\epsilon}^{j_{\nu_{2}}} J_{\nu}(t) d t
$$

and this, in turn, is positive for sufficiently small $\epsilon>0$, in view of R. G. Cooke's result [3] that

$$
\int_{0}^{j_{\nu 2}} J_{\nu}(t) d t>0, \quad \nu>-1 .
$$

Proof of (4)(ii). The same argument applies here to

$$
\int_{0}^{j_{\nu 2}} t^{-(\lambda+1 / 2)}\left[t^{1 / 2} J_{\nu}(t)\right] d t, \quad \lambda>-1 / 2, \quad \nu>1 / 2,
$$

in view of E. Makai's result [6] that

$$
\int_{0}^{j_{\nu 2}} t^{1 / 2} J_{\nu}(t) d t>0, \quad \nu>1 / 2 .
$$

${ }^{2}$ The results of [4] are summarized and extended in [5].

${ }^{3} \mathrm{Z}$. Ciesielski has mentioned that (4) (i) and (ii) can be inferred from the Cooke and Makai results, respectively, also via Theorem 1a of [1].

${ }^{4}$ Here the Bessel function of the first kind, $J_{\nu}(t)$, can be replaced by an arbitrary solution of the Bessel equation, say $\mathcal{C}_{\nu}(t)$, normalized so as to be positive for $t$ between zero and the first positive zero, with the parameters $\lambda, \nu$ restricted so as to insure convergence of the integral. This extension arises because [6], used in the proof of (4) (ii), covers this case. 
A simplified version (cf. [2]) of these proofs (the introduction of $\epsilon$ being superfluous) shows that

$$
(-1)^{p} \int_{j_{\nu p}}^{j_{\nu, p+2}} t^{-\lambda} J_{\nu}(t) d t>0\left\{\begin{aligned}
& \text { (i) } \lambda \geqq 0, \quad \nu>-1 \text { or } \\
& \text { (ii) } \quad \lambda>-1 / 2, \quad \nu>1 / 2,
\end{aligned}\right.
$$

where $j_{\nu p}$ is the $p$ th positive zero of $J_{\nu}(t), p=1,2, \cdots$.

4. Proof of the inequality. That $h_{\nu \lambda}(\theta)<1$ for $\lambda<\nu+1$, and either $\lambda \geqq 0, \nu>-1$ or $\lambda>-1 / 2, \nu>1 / 2$ follows at once by combining (4) and (5), since they imply the positivity of the numerator in (1), for all $\theta \neq 0$. The denominator is also positive (its value is contained in the proof of (3)).

To show that $h_{\nu \lambda}(\theta)>-1$ for appropriate $\nu, \lambda$, it suffices to show, as Wilf points out [7(a), p. 937], that the ratio of the integrals in (1) is less than 2. From (4) and (5) it is clear that the maximum of this ratio is achieved for $\theta=j_{\nu 1}$. But, for $\lambda>-1 / 2$, and all sufficiently large $\nu$, this ratio must be less than 2 , since the constant term in (3) is $1.2743521<2$.

Thus, (2) is established for all sufficiently large $\nu$, if $\lambda>-1 / 2$ and $\lambda<\nu+1$. In particular, (2) holds for $\lambda=1 / 2, \nu=2 k+3 / 2$, for all sufficiently large positive integers $k$, the case relevant to [7(a)].

\section{REFERENCES}

1. Z. Ciesielski, A note on some inequalites of Jensen's type, Ann. Polon. Math. 4 (1958), 269-274.

2. R. G. Cooke, Gibbs' phenomenon in Fourier-Bessel series and integrals, Proc. London Math. Soc. 27 (1928), 171-192.

3. - A monotonic property of Bessel functions, J. London Math. Soc. 12 (1937), 180-185.

4. Lee Lorch and Peter Szego, A singular integral whose kernel involves a Bessel function, Duke Math. J. 22 (1955), 407-418; Corrections and a remark, ibid., 24 (1957), 683.

5. - A singular integral whose kernel involves a Bessel function. II, Acta Math. Acad. Sci. Hungar. 13 (1962), 203-217.

6. E. Makai, On a monotonic property of certain Sturm-Liouville functions, Acta Math. Acad. Sci. Hungar. 3 (1952), 165-172.

7. (a) H. S. Wilf, The stability of smoothing by least squares, Proc. Amer. Math. Soc. 15 (1964), 933-937.

(b) Errata, Proc. Amer. Math. Soc. 17 (1966), p. 542.

University of Alberta, Edmonton and Ampex Corporation 